\title{
Novel SLC5A2 mutation contributes to familial renal glucosuria: Abnormal expression in renal tissues
}

\author{
LEI YU ${ }^{1}$, PING HOU ${ }^{2}$, GUO-PING LIU ${ }^{1}$ and HONG ZHANG ${ }^{2}$ \\ ${ }^{1}$ Renal Division, Inner Mongolia People's Hospital, Hohhot, Inner Mongolia 010017; \\ ${ }^{2}$ Renal Division, Peking University First Hospital, Peking University Institute of Nephrology, \\ Key Laboratory of Renal Disease, Ministry of Health of China, Beijing 100034, P.R. China
}

Received March 16, 2015; Accepted April 27, 2016

DOI: $10.3892 / \mathrm{etm} .2016 .3388$

\begin{abstract}
Familial renal glucosuria (FRG) is characterized by persistent glucosuria in the presence of normal serum glucose concentrations, while other impairments of tubular function are absent. Mutations in the sodium-glucose co-transporter 2 (SLC5A2) gene have been found to be responsible for FRG. However, direct evidence for the presence of SLC5A2 mutant in renal tissues is very rare. In previous studies, a non-sense mutation (c.1320 G>A:p.W440X) that would cause premature termination of the protein was found. However, the effects in the renal tissues were not reported. In the current study, a patient with FRG and a urinary glucose excretion rate of $8.3 \mathrm{~g} /$ day is described, for whom a novel missense mutation (c.1319G>A:p.W440X) was revealed by sequencing. Furthermore, in the immunofluorescence examination of a renal biopsy specimen, SLC5A2 was detected in the apical side of the proximal convoluted tubule, discontinuously decreased in comparison with that in normal and disease controls. The results imply that both wild-type SLC5A2 and mutant SLC5A2 with abnormal distribution were expressed in the renal tissues, and that the reduction of SLC5A2 expression and function were due to the c.1319G>A:p.W440X mutation. The current study provides valuable clues regarding the SLC5A2 molecule from genotype to phenotype in families affected by FRG.
\end{abstract}

\section{Introduction}

Sodium-glucose co-transporter 2 (SLC5A2) belongs to the $\mathrm{Na}^{+}$-glucose cotransporter family and acts as a critical

Correspondence to: $\mathrm{Dr}$ Lei Yu, Renal Division, Inner Mongolia People's Hospital, 20 Zhaowuda Road, Hohhot, Inner Mongolia 010017, P.R. China

E-mail: lanlife@163.com

Abbreviations: SLC5A2, sodium-glucose co-transporter 2; FRG, familial renal glucosuria

Key words: expression, familial renal glucosuria, function, mutation, SLC5A2 molecule in the process of glucose re-absorption from urine in the proximal convoluted tubule (1). The SLC5A2 gene is mapped to $16 \mathrm{p} 11.2$ (2) with a $7.7-\mathrm{kb}$ nucleic acid, containing 14 exons, and encoding 672 amino acids. The SLC5A2 protein is located in the early proximal convoluted tubule, segment $\mathrm{S} 1$, and has a $\mathrm{Na}^{+}$-to-glucose coupling ratio of 1:1 (3). Mutations in SLC5A2 (OMIM: 182381) have been confirmed as being responsible for the vast majority of cases of familial renal glucosuria (4-6). However, an increased glucose excretion was not observed in all individuals heterozygous for a specific mutation, and even among family members with identical SLC5A2 mutations, only some had mild glucosuria (4-6). In previous studies, a homozygous mutation (a G to A transition at position 1320) of p.W440X within exon 11 was found in two patients $(4,7)$. The parents and relatives of the patients in those studies were found to be heterozygous for the mutation and exhibited almost no renal glucosuria. The present study is notable in that a patient with evident renal glucosuria ( $8.3 \mathrm{~g} /$ day) was found to have a heterozygous mutation of c.1319G>A:p.W440X. As direct evidence for SLC5A2 heterozygous mutation of p.W440X in renal tissues has not yet been obtained, the present study reports the identification of a novel heterozygous mutation in $S L C 5 A 2$ and further investigated the effect of the mutant $S L C 5 A 2$ gene on protein expression in renal tissues.

\section{Materials and methods}

Patient and control individuals. The patient was a 36-year-old woman who had persistent glucosuria with normal serum glucose and no other evidence of renal disease. Glucosuria was quantified by $24 \mathrm{~h}$ urine collection. A total of 100 healthy Chinese individuals (200 chromosomes) were included as controls. In the immunofluorescence analysis of renal biopsy specimens, normal renal tissue from tumor patients following partial nephrectomy and biopsy tissue from patients with minimal change disease and diabetic nephropathy were included as normal and disease controls, respectively.

The study protocol was approved by the Medical Ethics Committee of Inner Mongolia People's Hospital (Hohhot, China). Informed written consent was obtained from all participants prior to participation in the study. 
Mutation screening. Genomic DNA was extracted by salting out from peripheral white blood cells. The entire coding region and adjacent intronic segments of the SLC5A2 gene were screened for mutation by direct sequencing of polymerase chain reaction (PCR) products, generated with primers described previously (8). The genomic DNA reference sequences of SLC5A2 (NC_000016.10, OMIM: 182381, Gene ID: 6524) were obtained from the Entrez gene database. The 200 control chromosomes were tested by direct sequencing of PCR products to ensure that these mutations do not represent common polymorphisms.

Immunofluorescence studies on renal biopsy specimen. Renal specimens were evaluated by routine methods using indirect immunofluorescence, and light and electron microscopy. SLC5A2 expression in the renal biopsy specimens was detected by immunofluorescence staining as described in our previous study (9). In brief, renal biopsy specimens were incubated overnight at $4{ }^{\circ} \mathrm{C}$ with a goat anti-human polyclonal antibody targeting SLC5A2 (1:50; sc-47404; Santa Cruz Biotechnology, Inc., Dallas, TX, USA), and then a fluorescein isothiocyanate-labeled donkey anti-goat IgG antibody (1:200; sc-2024; Santa Cruz Biotechnology, Inc.) for $30 \mathrm{~min}$ at $37^{\circ} \mathrm{C}$. Normal renal tissue from tumor patients following partial nephrectomy was included as a normal control. Biopsy tissue from patients with minimal change disease and diabetic nephropathy was included as a disease control. Specific binding was defined by replacing primary antibody with $1 \%$ bovine serum albumin (Sigma-Aldrich, St. Louis, MO, USA). Sections were observed using a confocal microscope (Olympus FV1000; Olympus Corporation, Tokyo, Japan).

\section{Results}

Clinical characterization of the patient. The patient was a 36-year-old woman who was referred to the renal division because of repeated glucosuria. The patient had no polyuria, polydipsia or weight loss. A review of systems and the physical examination were completely normal. The patient's blood pressure was $110 / 80 \mathrm{mmHg}$ and body weight was $50 \mathrm{~kg}$. Fasting plasma glucose $(4.84 \mathrm{mmol} / \mathrm{l})$, albumin $(46.7 \mathrm{~g} / \mathrm{l})$, creatinine $(71.00 \mu \mathrm{mol} / \mathrm{l})$, sodium $(142.00 \mathrm{mmol} / \mathrm{l})$, chloride (106.0 mmol/l), potassium (4.40 mmol/l), calcium $(2.34 \mathrm{mmol} / \mathrm{l})$, phosphate $(0.96 \mathrm{mmol} / \mathrm{l})$, magnesium $(0.82 \mathrm{mmol} / \mathrm{l})$, bicarbonate (26.30 mmol/l), uric acid $(225 \mu \mathrm{mol} / \mathrm{l})$ and glycated hemoglobin $(5.7 \%)$ levels were all within the normal range. Routine urinary analysis revealed $2^{+}$to $4^{+}$results for glucose with no other abnormalities. A quantitative test for urine glucose provided a result of $8.3 \mathrm{~g} / 24 \mathrm{~h}$. Urinary glucose excretion in family members was confirmed by qualitative test. The father and a uncle of patient had increased urinary glucose excretions of $1-2^{+}$. No other marked abnormality was detected in the urine and blood biochemistry of family members (Fig. 1A). Secondly, the patient was characterized by persistent glucosuria despite normal serum glucose and the absence of overt tubular dysfunction. Routine analysis of renal specimens using indirect immunofluorescence, and light and electron microscopy revealed no apparent glomerular or tubulointerstitial lesions. Furthermore, a novel mutation was identified in the patient. From above all, the patient could be diagnosis of familial renal glucosuria (FRG) securely.
Identification of a novel mutation in the patient. Using mutation screening of genomic DNA, a novel missense mutation was identified in the patient (c.1319G>A: p.W440X, Fig. 1). This identified mutation was not detected in any of the 200 chromosomes derived from 100 healthy, unrelated individuals, indicating that the mutation does not represent a common polymorphism.

Identified mutation alters SLC5A2 expression in the kidney. Renal biopsy in the patient with familial renal glucosuria (FRG) revealed no apparent glomerular or tubulointerstitial lesions when examined by immunofluorescence, light and electron microscopy. However, the expression of SLC5A2 in the apical side of the proximal convoluted tubule was discontinuously decreased in comparison with that in both normal and disease controls (patients with minimal change disease or diabetic nephropathy; Fig. 2).

\section{Discussion}

Glucose is the fuel that provides energy for normal activity in humans, and the major source of glucose is carbohydrates in food. The kidneys help to keep blood glucose levels normal by reabsorbing $\sim 180 \mathrm{~g}$ glucose per day through filtration in the proximal tubules (1). SLC5A2 is responsible for the active transport of glucose across the brush border membrane, and is expressed almost exclusively in the kidney, accounting for the bulk of glucose reabsorption (1). Studies report that SLC5A2 mutations are involved in FRG (9-12). The long-term outcome of patients with FRG is very good, and so SLC5A2 inhibitors have been the subject of particular attention in the search for potential new drug targets for the treatment of diabetes $(13,14)$. However, safety issues have hindered the development of SLC5A2 inhibitors (15). Research on patients with FRG may bring about a breakthrough in the study of the treatment of diabetes. In previous studies, although a homozygous mutation ( $G$ to A transition at position 1320) within exon 11 was found in two patients, the effect of the mutation, especially the heterozygous mutation of p.W440X, on the expression of SLC5A2 in the kidney is not yet fully known (4,7). In the present study, to exclude tubular impairments or other renal diseases that could cause glucosuria, the patient agreed to undergo a renal biopsy. This revealed no apparent glomerular or tubulointerstitial lesions under immunofluorescence, light and electron microscopy.

A novel SLC5A2 mutation of c.1319G>A:p.W440X was found in the present patient with FRG. The data reveal that a patient with a heterozygous mutation had clinical manifestations (urinary glucose excretion rate, $8.3 \mathrm{~g} /$ day), which implies that the mutation causes clinically relevant SLC5A2 dysfunction. In the immunofluorescence examination of SLC5A2 in the renal biopsy specimen, SLC5A2 was detected in the apical side of the proximal convoluted tubule, discontinuously decreased in comparison with both normal and disease controls. Therefore, the wild-type SLC5A2 exhibits normal expression and the mutation appears to decrease the expression of SLC5A2 in the apical side of the proximal convoluted tubule. Therefore, the mutation may affect transport activity by altering protein processing and impairing protein insertion into the plasma membrane. Furthermore, it implies that 
A

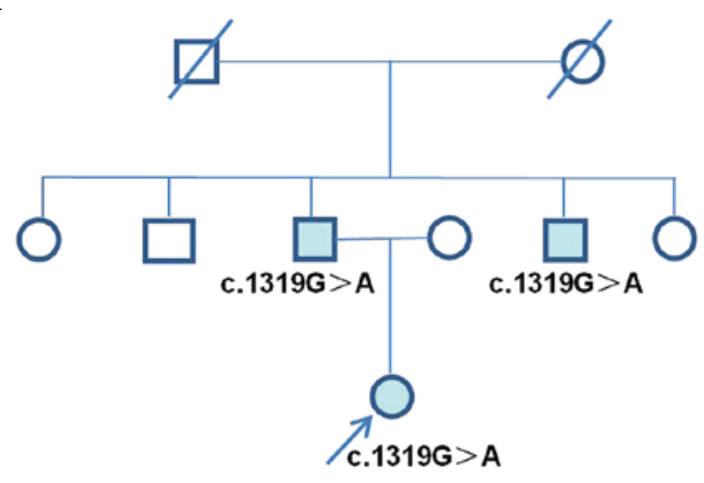

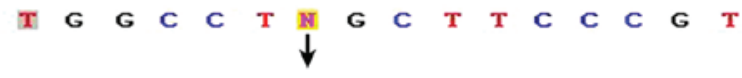

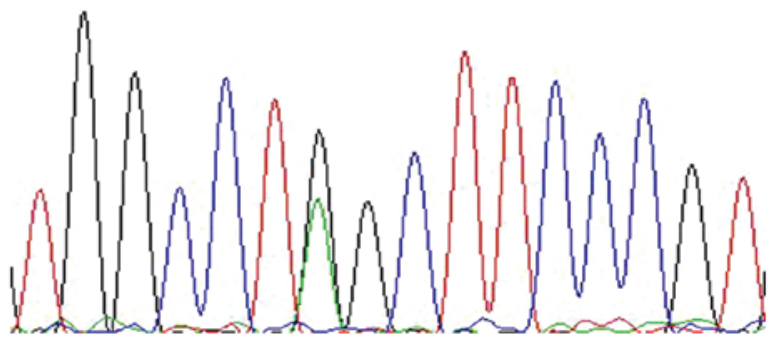

c.1319G $>$ A

Figure 1. Familial renal glucosuria patient carried a novel mutation (c.1319G>A:p.W440X). Nucleotides are numbered according to sodium-glucose o-transporter 2 (SLC5A2) cDNA sequence. (A) Familial renal glucosuria pedigree carries the novel mutation. Circles: Females; squares: males; blue coloring: With increased urinary glucose excretion; strike through: death. (B) Mutation screening of genomic DNA and a novel missense mutation was identified in the patient.
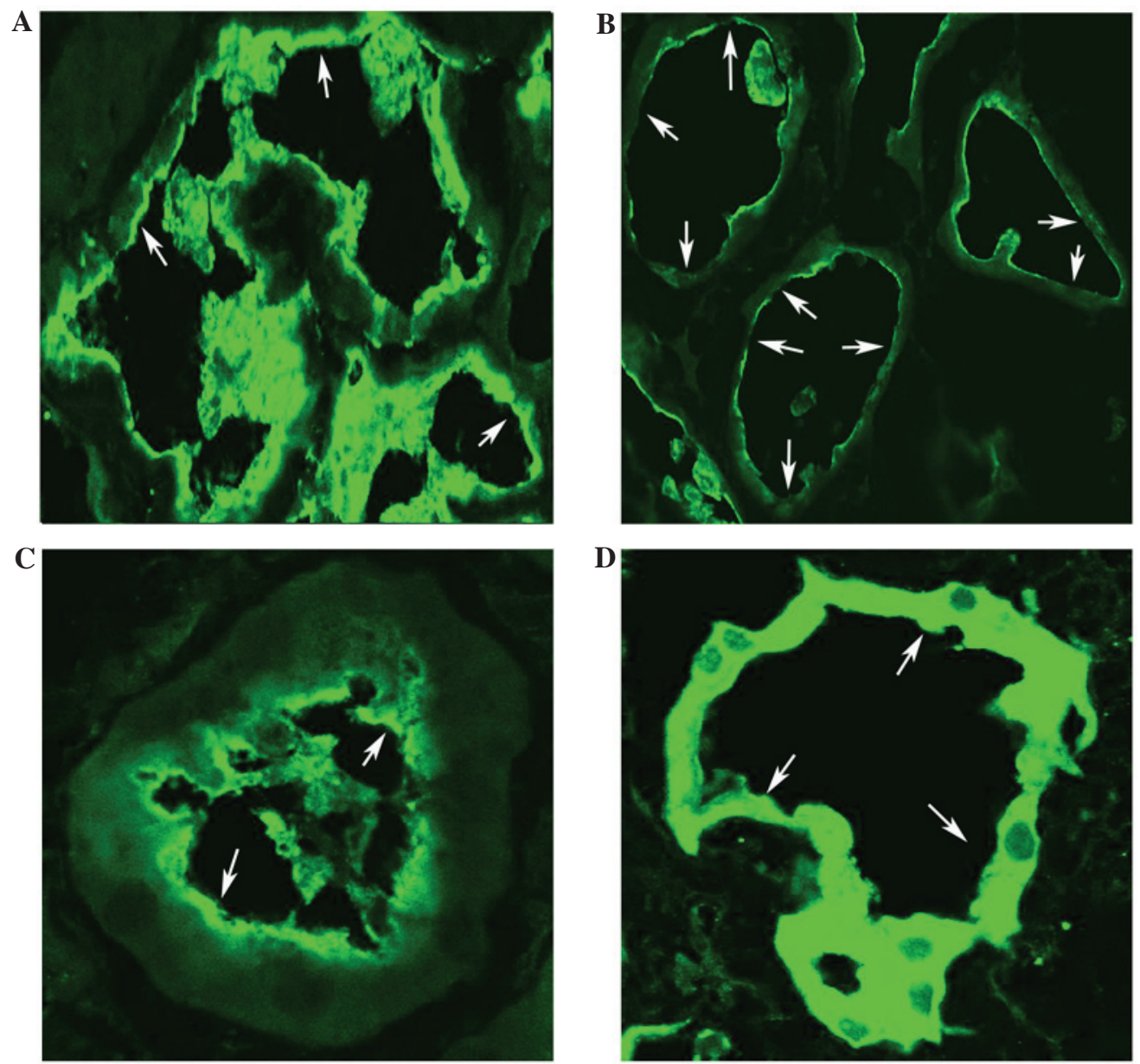

Figure 2. Immunofluorescence of SLC5A2 in kidney specimens. sodium-glucose co-transporter 2 (SLC5A2 in (A) normal kidney, (B) the patient with familial renal glucosuria (FRG), (C) a patient with minimal change disease and (D) a patient with diabetic nephropathy (magnification, x300). SLC5A2 was distributed continuously and mainly in the apical side of the proximal convoluted tubule in normal and disease controls. In the FRG patient, SLC5A2 in the apical side of the proximal convoluted tubule was discontinuously decreased in comparison with both normal and disease controls.

abnormal distribution of mutant SLC5A2 might play a key role in affecting transport activity. From the results, the inheritance of renal glucosuria can be described as a codominant trait as a whole, and it may be inferred that variable penetrance may be associated with the compensatory capacity of wild-type SLC5A2. To the best of our knowledge, the altered SLC5A2 expression caused by heterozygous mutation of p.W440X has not been reported previously in human subjects. This observation may shed light on the function of SLC5A2; however, further analysis is required.

In summary, a novel SLC5A2 mutation was identified in a Chinese patient with FRG, and SLC5A2 was observed to be 
discontinuously decreased in the apical side of the proximal convoluted tubule in the patient on renal biopsy. The mutant SLC5A2 proteins may have significantly lowered SLC5A2 expression in the apical side of the proximal convoluted tubule. This study provides valuable information concerning the role of the SLC5A2 molecule from genotype to phenotype.

\section{Acknowledgements}

This study was funded by the National Natural Science Foundation of China (grant no. 81160087), the Natural Science Fund of Inner Mongolia Autonomous Region (grant no. 2010BS1102), 2015 Science and Technology Planning Project of Inner Mongolia Autonomous Region (grant no. 201502107) and the Fund Program of Inner Mongolia People's Hospital (grant no. 201510).

\section{References}

1. Wright EM, Hirayama BA and Loo DF: Active sugar transport in health and disease. J Intern Med 261: 32-43, 2007.

2. Wells RG, Mohandas TK and Hediger MA: Localization of the $\mathrm{Na}^{+} /$glucose cotransporter gene SGLT2 to human chromosome 16 close to the centromere. Genomics 17: 787-789, 1993.

3. Kanai Y, Lee WS, You G, Brown D and Hediger MA: The human kidney low affinity $\mathrm{Na}(+) /$ glucose cotransporter SGLT2: Delineation of the major renal reabsorptive mechanism for D-glucose. J Clin Invest 93: 397-404, 1994.

4. Santer R, Kinner M, Lassen CL, Schneppenheim R, Eggert P, Bald M, Brodehl J, Daschner M, Ehrich JH, Kemper M, et al: Molecular analysis of the SGLT2 gene in patients with renal glucosuria. J Am Soc Nephrol 14: 2873-2882, 2003.
5. Calado J, Sznajer Y, Metzger D, Rita A, Hogan MC, Kattamis A, Scharf M, Tasic V, Greil J, Brinkert F, et al: Twenty-one additional cases of familial renal glucosuria: Absence of genetic heterogeneity, high prevalence of private mutations and further evidence of volume depletion. Nephrol Dial Transplant 23: 3874-3879, 2008

6. Lee H, Han KH, Park HW, Shin JI, Kim CJ, Namgung MK, Kim KH, Koo JW, Chung WY, Lee DY, et al: Familial renal glucosuria: A clinicogenetic study of 23 additional cases. Pediatr Nephrol 27: 1091-1095, 2012.

7. Van den Heuvel LP, Assink K, Willemsen M and Monnens L: Autosomal recessive renal glucosuria attributable to a mutation in the sodium glucose cotransporter (SGLT2). Hum Genet 111: 544-547, 2002.

8. Magen D, Sprecher E, Zelikovic I and Skorecki K: A novel missense mutation in SLC5A2 encoding SGLT2 underlies autosomal-recessive renal glucosuria and aminoaciduria. Kidney Int 67: 34-41, 2005.

9. Yu L, Hou P, Lv JC, Liu GP and Zhang H: A novel sodium-glucose co-transporter 2 gene (SGLT2) mutation contributes to the abnormal expression of SGLT2 in renal tissues in familial renal glucosuria. Int Urol Nephrol 46: 2237-2238, 2014.

10. Yu L, Lv JC, Zhou XJ, Zhu L, Hou P and Zhang H: Abnormal expression and dysfunction of novel SGLT2 mutations identified in familial renal glucosuria patients. Hum Genet 129: 335-344, 2011.

11. Lee YW: Clinical and genetic analysis in a patient with primary renal glucosuria: Identification of a novel mutation in the SLC5A2 gene. Exp Ther Med 6: 1532-1534, 2013.

12. Yu L, Hou P, Lv JC, Liu GP and Zhang H: Novel SLC5A2 variants contribute to renal glucosuria in chinese families: Abnormal expression and dysfunction of variant SLC5A2. Hum Mutat 36: 79-86, 2015.

13. Scholl-Bürgi S, Santer R and Ehrich JH: Long-term outcome of renal glucosuria type 0 : The original patient and his natural history. Nephrol Dial Transplant 19: 2394-2396, 2004.

14. Isaji M: Sodium-glucose cotransporter inhibitors for diabetes. Curr Opin Investig Drugs 8: 285-292, 2007.

15. Ledford H: Diabetes drugs ride a bumpy road. Nature 504: 198 , 2013. 